\title{
Signal and Noise
}

\section{OVERVIEW}

Let us very briefly review the basics of signal detection. This will help set the stage of the signal behavior in MRI. First, a transverse component is achieved by the application of an RF pulse. The resulting transverse magnetization creates an electromotive force (emf) in any coil through which the spin's magnetic flux sweeps, a consequence of Faraday's law of induction (see UNIT B2.1). When all the amplification factors and relaxation times are ignored, we find:

$$
\text { signal for homogeneous object } \propto \omega_{0} M_{0} \mathcal{B}_{\perp} V_{\text {sample }}
$$

where $\omega_{0}$ is Larmor frequency, $M_{0}$ is bulk magnetization, $\mathcal{B}_{\perp}$ is the transverse field amplitude produced by the receive coil when a unit current is passed through it, and $V_{\text {sample }}$ is the volume of homogeneous sample.

In general, the MR signal is detected in quadrature (i.e., it is measured in two orthogonal channels). The signal is then demodulated with respect to the Larmor frequency and digitized. The digitized MR signals are stored as real and imaginary raw data, corresponding to each channel. The conventional magnitude and phase MR images are reconstructed from the complex raw data using a complex Fourier transform.

Akin to all physical measurements, the measured MR signal also includes noise. The noise in MR generally derives from the random fluctuations in the receive coil electronics and from the sample. Even though there are other sources of signal fluctuations such as digitization noise and pseudo-random ghosting due to moving spins, these sources are minimized in an ideal experiment. The noise corrupts the image and, if it gets too large, degrades its value significantly.

The measured MR signal in $k$-space can be thought of as the sum of the true signal $s(k)$ with white noise $\varepsilon(k)$ added to it to give the noisy measured signal $s_{\mathrm{m}}(k)$ :

$$
s_{\mathrm{m}}(k)=s(k)+\varepsilon(k)
$$

The additive white noise $(\varepsilon(k))$ is typically characterized as being Gaussian distributed with zero mean and variance $\sigma_{\mathrm{m}}^{2}$ in both real and imaginary channels. Similarly, the noise in real and imaginary MR images obtained from the complex $k$-space data via Fourier transform is also Gaussian-distributed with zero mean and variance $\sigma_{0}^{2}=\sigma^{2}{ }_{\mathrm{m}} / N$ where $N$ is the number of sampled points.

Generally, for larger signal relative to noise, the noise is still roughly Gaussian-distributed (see Fig. B6.1.1A) with zero mean and variance $\sigma_{0}^{2}$, assuming signal magnitude is at least three times larger than the noise standard deviation. The noise outside of an magnitude image, in the background, is Raleigh-distributed (see Fig. B6.1.1B) with mean $1.253 \sigma_{0}$ and standard deviation $0.655 \sigma_{0}$, respectively (Henkelman, 1985). One way to measure and visualize the variance is to perform the same experiment many times and calculate the variance on a pixel-by-pixel basis. The phase image has similar noise characteristics, being also roughly Gaussian distributed but with zero mean and variance of $\sigma_{\mathrm{P}}^{2}$. The noise 
A

A Gaussian distribution

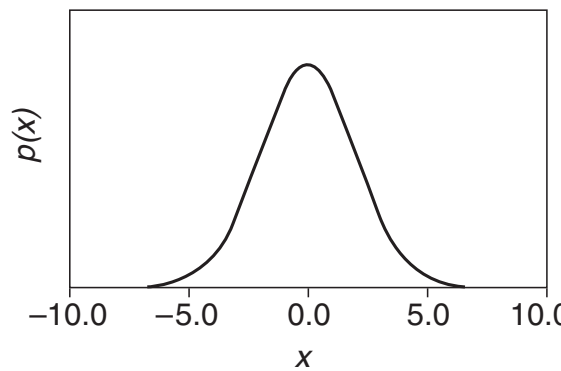

B

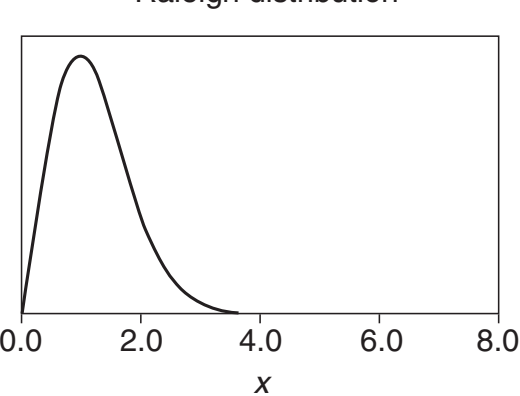

Figure B6.1.1 Noise distributions from a magnitude image (A) inside the image (Gaussian distribution with zero mean and standard deviation 2) and (B) in the background (Raleigh distribution with mean equal to 1.253) are shown.

variance in a phase image inside the object is related to the noise variance of the magnitude image as follows:

$$
\sigma_{\mathrm{P}}^{2}=\frac{\sigma_{0}^{2}}{\rho_{\mathrm{m}}^{2}}
$$

where $\rho_{\mathrm{m}}$ is the measured amplitude in the magnitude image.

\section{TECHNICAL DISCUSSION}

\section{The Voxel Signal}

After the transverse magnetization has been encoded for spatial information, the general $k$-space signal representation is given by:

$$
s(\vec{k})=\int_{V} d^{3} r \rho(\vec{r}) e^{-i 2 \pi \vec{k} \cdot \vec{r}}
$$

When the signal expression in Equation B6.1.4 is written for the 3-D Fourier imaging case, it becomes:

$$
s\left(k_{x}, k_{y}, k_{z}\right)=\iiint d x d y d z \rho(x, y, z) e^{-i 2 \pi\left(k_{x} x+k_{y} y+k_{z} z\right)}
$$

The data are assumed to be sampled at a set of points in 3-D $k$-space. These points are separated by $\Delta k_{x}, \Delta k_{y}$, and $\Delta k_{z}$, respectively, in the three orthogonal $k$-space directions, and cover the range defined by the following set of integers:

$p^{\prime}, q^{\prime}$, and $r^{\prime} \in\left(-N_{x}, N_{x}-1\right),\left(-N_{y}, N_{y}-1\right),\left(-N_{z}, N_{z}-1\right)$.

The image is reconstructed by applying a discrete inverse Fourier transform to the set of data represented by $s_{\mathrm{m}}\left(p^{\prime} \Delta k_{x}, q^{\prime} \Delta k_{y}, r^{\prime} \Delta k_{z}\right)$. Likewise, the image is represented by $\hat{\rho}_{\mathrm{m}}(p \Delta x$, $q \Delta y, r \Delta z$ ) where $p, q$, and $r$ typically cover the same range as $p^{\prime}, q^{\prime}$, and $r^{\prime}$. The signal at a given point in the image is then given by the inverse Fourier transform of $s(\vec{k})$ : 


$$
\hat{\rho}_{m}(p \Delta x, q \Delta y, r \Delta z)=\frac{1}{N_{x} N_{y} N_{z}} \sum_{p^{\prime}, q^{\prime}, r^{\prime}} s\left(p^{\prime} \Delta k_{x}, q^{\prime} \Delta k_{y}, r^{\prime} \Delta k_{z}\right) e^{i 2 \pi\left(\frac{p p^{\prime}}{N_{x}}+\frac{q q^{\prime}}{N_{y}}+\frac{r r^{\prime}}{N_{z}}\right)}
$$

The effective spin-density $\hat{\rho}_{\mathrm{m}}(p \Delta x, q \Delta y, r \Delta z)$ is also known as the "voxel signal" $S(\vec{r})$ since it is the signal which will be represented in the volume element $\Delta x \Delta y \Delta z$ at position ( $p \Delta x$, $q \Delta y, r \Delta z)$ in the reconstructed image. Recall that $\hat{\rho}_{\mathrm{m}}(p \Delta x, q \Delta y, r \Delta z)$ often not only represents the transverse magnetization within the voxel volume but also contains a handful of scaling factors and relaxation parameters. Here, the use of effective spin density is no more appropriate than using transverse magnetization or number of spins in a voxel. It has been used in this way because all voxels in a conventional Fourier imaging approach have the same dimensions, and, hence, the actual spin density times this volume has the same relative behavior from voxel to voxel as what we have called, for simplicity, effective spin density.

The voxel size represents the area under the point spread function normalized to the zero value of the point spread function. As a result, whenever the spatial resolution is improved by reducing $\Delta x, \Delta y$, or $\Delta z$, the total contribution to the voxel signal is reduced proportionately; for example, if $\Delta x$ is halved, $\hat{\rho}_{\mathrm{m}}(p \Delta x, q \Delta y, r \Delta z)$ is also halved; therefore, in general:

$$
\hat{\rho}_{\mathrm{m}}(p \Delta x, q \Delta y, r \Delta z) \propto \Delta x \Delta y \Delta z \equiv \text { voxel volume } \equiv V_{\text {voxel }}
$$

It can be shown that the peak signal for a homogeneous sample (at $t=0$ in a free induction decay experiment, or at $t=T_{\mathrm{E}}$ for a gradient echo or spin echo imaging experiment) when transverse signal decay and amplification factors are neglected is given by:

$$
\text { signal for homogeneous object } \propto \omega_{0} M_{0} \mathcal{B}_{\perp} V_{\text {sample }}
$$

where $\mathcal{B}_{\perp}$ is the transverse field amplitude produced by the receive coil when a unit current is passed through it. Since the voxel is assumed to be a small homogeneous volume element in these discussions, Equation B6.1.8 can be rewritten for proton imaging as:

$$
\begin{aligned}
S & \equiv \hat{\rho}_{\mathrm{m}}(p \Delta x, q \Delta y, r \Delta z) \\
& \propto \omega_{0} \cdot \frac{\frac{1}{2} \cdot \frac{3}{2} \cdot \gamma^{2} \hbar^{2}}{3 k T} \cdot B_{0} \cdot \mathcal{B}_{\perp}(p \Delta x, q \Delta y, r \Delta z) \cdot \Delta x \Delta y \Delta z \\
& \propto \frac{\gamma^{3} \hbar^{2}}{4 k T} \cdot B_{0}^{2} \cdot \mathcal{B}_{\perp}(p \Delta x, q \Delta y, r \Delta z) \cdot \mathrm{V}_{\text {voxel }}
\end{aligned}
$$

It is important to note the different dependencies in Equation B6.1.9. Of interest is the direct dependence on the voxel volume, on $\mathcal{B}_{\perp}$ and on $B^{2}{ }_{0}$, as well as the inverse dependence on the sample temperature.

\section{The Noise in MRI}

The variance of the fluctuating noise voltage is presented here, without proof (Kittel, 1969) to be: 


$$
\operatorname{var}\left(\mathrm{emf}_{\text {noise }}\right) \equiv \sigma_{\text {thermal }}^{2} \propto \overline{\left(\mathrm{emf}_{\text {noise }}-\overline{\mathrm{emf}_{\text {noise }}}\right)^{2}}=4 k T \cdot R_{\text {eff }} \cdot \mathrm{BW}
$$

where the horizontal bar over a value implies an average value, $R_{\text {eff }}$ is the effective resistance of the coil loaded by the body, and BW is the bandwidth of the noise-voltage detecting system. In MRI, both the signal and the noise are detected by the receive coil and the bandwidth $\left(\mathrm{BW}_{\text {read }}\right.$ ) of reception is determined by the cutoff frequency of the analog low-pass filter which is the bandwidth in the read direction.

The random thermal fluctuations in the measured signal are called "white" fluctuations because they are characterized by equal expected noise power components at all frequencies within the readout bandwidth. The noise "variance" of the body and coil together is the sum of variances since these statistical processes are independent, leading to:

$$
\sigma_{\text {thermal }}^{2}(\vec{k})=\sigma_{\text {body }}^{2}(\vec{k})+\sigma_{\text {coil }}^{2}(\vec{k})+\sigma_{\text {electronics }}^{2}(\vec{k})
$$

for all $k$-space values. For all further subsections, the shorthand notation $\sigma^{2}$ is typically used in place of $\sigma_{\text {thermal }}^{2}$ where the subscript " $m$ " connotes "measured." It is seen that an effective or total resistance can be inserted into Equation B6.1.10 representing the sum of the individual components:

$$
R_{\text {eff }}=R_{\text {body }}+R_{\text {coil }}+R_{\text {electronics }}
$$

\section{Dependence of the noise on imaging parameters}

Just as the voxel signal depends on the voxel volume which is an imaging parameter, the variance of the voxel signal due to noise is also found to depend on the choice of imaging parameters through the discrete inverse Fourier transform. As before, the measured $k$-space signal can be thought of as the true $k$-space signal $s(k)$ with white noise $\varepsilon(k)$ added to it to give the noisy measured signal $s_{\mathrm{m}}(k)$ :

$$
s_{\mathrm{m}}(k)=s(k)+\varepsilon(k)
$$

For this characterization, the noise autocorrelation function, which is an indication of how the value of $\varepsilon(k)$ at one time is statistically related to that at another time, is defined as:

$$
\left.R_{\varepsilon}(\tau) \equiv \overline{\varepsilon\left(k_{p}\right) \varepsilon^{*}\left(k_{q}\right)}\right|_{\tau \equiv\left(k_{p}-k_{q}\right)}
$$

and the Fourier transform of $R_{\varepsilon}(\tau), r_{\eta}(f)$, is known as the spectral density. $R_{\varepsilon}(\tau)$ is given by an impulse of strength $\sigma_{\mathrm{m}}^{2}-$ i.e.:

$$
R_{\varepsilon}(\tau)=\sigma_{m}^{2} \delta(\tau)
$$

and the spectral density is seen to be white since:

$$
\begin{aligned}
r_{\eta}(f) & \equiv \int d \tau R_{\varepsilon}(\tau) e^{-i 2 \pi f \tau} \\
& =\sigma_{\mathrm{m}}^{2}
\end{aligned}
$$


Equation B6.1.14 to Equation B6.1.16 simply state that the noise in the two orthogonal channels (the real and imaginary channels) are not correlated with each other. The above expression is valid for continuous $k$-space data measurement. In the discrete case:

$$
\overline{\varepsilon\left(k_{p}\right) \varepsilon^{*}\left(k_{q}\right)}=\sigma_{\mathrm{m}}^{2} \delta_{p q}
$$

where now $k_{p} \equiv p \Delta k$ and $k_{q}=q \Delta k$ and the Dirac delta $(\delta(\tau))$ is replaced by a Kronecker delta, $\delta_{p q}$. As before, this implies that any two white noise samples are uncorrelated and the expected noise power is $\sigma_{\mathrm{m}}^{2}$.

White noise is typically characterized as being Gaussian (or normal) distributed with zero mean and variance $\sigma_{\mathrm{m}}^{2}$. This distribution is denoted by $\mathcal{N}\left(0, \sigma_{\mathrm{m}}\right)$. Two Gaussian distributed random variables which are uncorrelated are also independent.

This information makes it possible to evaluate the statistical properties of the noise in the image domain. Using the definition of the discrete inverse Fourier transform acting on $\varepsilon(k)$, the white noise transforms to:

$$
\eta(p \Delta x)=\frac{1}{N} \sum_{p^{\prime}} \varepsilon\left(p^{\prime} \Delta k\right) e^{i 2 \pi p^{\prime} \Delta k \Delta \Delta x}
$$

in the image domain. In Equation B6.1.18, $N$ is the total number of samples and $\Delta x$ is the voxel size along the read direction. Recall that the $k$-space variable $\varepsilon\left(p^{\prime} \Delta k\right)$ is assumed to have a distribution of the form $\mathcal{N}\left(0, \sigma_{\mathrm{m}}\right)$. Taking the expectation of both sides, we get:

$$
\begin{aligned}
\overline{\eta(p \Delta x)} & =\frac{1}{N} \sum_{p^{\prime}} \overline{\varepsilon\left(p^{\prime} \Delta k\right)} e^{i 2 \pi p^{\prime} \Delta k \Delta \Delta x} \\
& =0
\end{aligned}
$$

Taking the variance of both sides yields a result independent of $p$ :

$$
\begin{aligned}
\operatorname{var}(\eta(p \Delta x)) \equiv \sigma_{0}^{2}(p \Delta x) & =\frac{1}{N^{2}} \sum_{p^{\prime}} \sum_{q^{\prime}} \overline{\varepsilon\left(p^{\prime} \Delta k\right) \varepsilon^{*}\left(q^{\prime} \Delta k\right)} e^{i 2 \pi p \Delta x\left(p^{\prime} \Delta k-q^{\prime} \Delta k\right)} \\
& =\frac{\sigma_{\mathrm{m}}^{2}}{N^{2}} \sum_{p^{\prime}} \sum_{q^{\prime}} \delta_{p^{\prime} q^{\prime}} e^{i 2 \pi p \Delta x\left(p^{\prime} \Delta k-q^{\prime} \Delta k\right)} \\
& =\frac{\sigma_{\mathrm{m}}^{2}}{N}
\end{aligned}
$$

where the independence of the random variables $\varepsilon\left(p^{\prime} \Delta k\right)$ and $\varepsilon\left(q^{\prime} \Delta k\right)$ as defined in Equation B6.1.17 is used.

Note that $\sigma_{\mathrm{m}}^{2}$ is the measured variance of any point in $k$-space, while $\sigma_{0}^{2}$ indicates the noise variance in the image domain. Hence, the variance measured in any voxel in the image domain is $N$ times smaller than that in the detected signal and is the same for all voxels. It is also common to quote the noise as the standard deviation $\sigma_{0}(p \Delta x)$. In conclusion, from Equation B6.1.20, as $N$ increases to $a N(a>1)$, noise standard deviation $\left(\sigma_{0}(p \Delta x)\right)$ decreases by the factor $\sqrt{a}$.

Signal, Noise, and Contrast

B6.1.5 
The image can now be rewritten, due to the linearity of the Fourier transform, as:

$$
\hat{\rho}_{\mathrm{m}}(p \Delta x)=\hat{\rho}_{\mathrm{m}, 0}(p \Delta x)+\eta(p \Delta x)
$$

where $\eta(p \Delta x)$ has zero mean and variance $\sigma_{0}^{2}$. In Equation B6.1.21, $\hat{\rho}_{\mathrm{m}, 0}$ represents the pristine image without any noise.

A simple and instructive example of these statistical concepts is illustrated by setting $p=$ 0 and examining the image at the origin:

$$
\begin{aligned}
\hat{\rho}_{\mathrm{m}}(0) & =\frac{1}{N} \sum_{p^{\prime}} s\left(p^{\prime} \Delta k\right)+\frac{1}{N} \sum_{p^{\prime}} \varepsilon\left(p^{\prime} \Delta k\right) \\
& =\overline{s(k)}+\eta(0)
\end{aligned}
$$

The first term is nothing more than the average over all $k$-space values for $s(k)$ for the pristine data while the second term represents the noise in the image domain. Since the expectation of each term $\varepsilon\left(p^{\prime} \Delta k\right)$ is zero so is that for $\eta(0)$ (in agreement with Equation B6.1.19). And the variance of $\eta(0)$ is immediately:

$$
\sigma_{0}^{2}=\frac{1}{N} \sigma_{\mathrm{m}}^{2}
$$

since the $\varepsilon\left(p^{\prime} \Delta k\right)$ are independent.

The standard deviation is then:

$$
\sigma_{0}=\frac{\sigma_{\mathrm{m}}}{\sqrt{N}}
$$

When half Fourier methods are used, $N$ is reduced to $N / 2$ and the noise gets larger by a factor of $\sqrt{2}$. That is, the sacrifice for a faster acquisition time in this case is the loss of SNR.

The generalization of Equation B6.1.20 to two dimensions (2-D) gives:

$$
\left.\sigma_{0}^{2}\right|_{2 D}=\frac{\sigma_{\mathrm{m}}^{2}}{N_{\mathrm{x}} N_{\mathrm{y}}}
$$

and to three dimensions (3-D) gives:

$$
\left.\sigma_{0}^{2}\right|_{3 D}=\frac{\sigma_{\mathrm{m}}^{2}}{N_{x} N_{y} N_{z}}
$$

Equations B6.1.25 and B6.1.26 indicate that the noise standard deviation is reduced significantly in a 3-D imaging experiment.

We have just shown above that when resolution is changed by increasing the number of sampling points by a factor $b$, then the noise standard deviation drops by $\sqrt{b}$-i.e, the noise is reduced. Now, in the case when partial Fourier imaging is used, the resolution actually stays the same even though the number of points is decreased by a factor of two relative to the usual symmetric acquisition; therefore, the signal per voxel does not change, but the noise standard deviation does. It now goes up by $\sqrt{2}$ because there are essentially fewer points to average out the noise effects. 


\section{Noise in Background}

The complex signal can be written as:

$$
\hat{\rho}=R+i \mathrm{I}
$$

or as:

$$
\hat{\rho}=\rho_{\mathrm{m}} e^{i \phi}
$$

where $\rho_{\mathrm{m}}$ is the magnitude signal. Let $\rho_{\mathrm{m}}$ in the absence of noise be $a$. If the noise-free phase is $\hat{\phi}$ then the real and imaginary parts, $R$ and $I$, respectively, of $\hat{\rho}$ are:

$$
\begin{aligned}
& R=a \cos \hat{\phi}+\eta_{1} \\
& \mathrm{I}=a \sin \hat{\phi}+\eta_{2}
\end{aligned}
$$

where $R$ and $I$ are the real and imaginary parts of the reconstructed complex voxel signal, respectively, and $\eta_{1}, \eta_{2}$ are noise samples from a Gaussian distribution with mean zero and variance $\sigma_{0}^{2}$.

The magnitude representation of the background noise $(a=0)$ is given by:

$$
\hat{\rho}_{b}=\sqrt{R^{2}+I^{2}}=\sqrt{\eta_{1}^{2}+\eta_{2}^{2}}
$$

Then, it can be shown that the noise distribution in the background (assuming no signal is available) has a Rayleigh distribution (see Papoulis, 1991) and is given by:

$$
p\left(\rho_{b}\right)=\frac{\rho_{\mathrm{b}}}{\sigma_{\mathrm{b}}^{2}} e^{-\rho_{b}^{2} / 2 \sigma_{\mathrm{b}}^{2}}
$$

It is possible to estimate the standard deviation of the noise in the magnitude image inside the object from the experimentally measured background noise distribution. The mean and standard deviation of the Rayleigh distribution is related to the standard deviation of the Gaussian distribution as $1.253 \sigma_{0}$ and $0.655 \sigma_{0}$, respectively (Henkelman, 1985).

Phase images offer some fascinating ways to enhance information about certain features in MR, and phase is commonly used in flow imaging to estimate velocities. Since taking the phase is also a nonlinear operation the noise distribution of the phase is no longer Gaussian. It can be shown that the probability distribution of the noise in the background of a phase image can be given by (Gudbjartson and Patz, 1995): 


$$
p(\phi)=\frac{1}{2 \pi} \text { for }-\pi<\phi<\pi
$$

Equation B6.1.33 indicates that the noise in the background in a phase image is uniformly distributed between $-\pi$ and $\pi$.

In summary, the noise in the background of the magnitude and phase images has Rayleigh and uniform distributions, respectively. The background noise distribution is particularly important to estimate the original Gaussian distribution parameters in a magnitude image.

\section{Noise Variance in a Magnitude Image Inside the Object}

The mean values of real and imaginary signals, $\bar{R}$ and $\bar{I}$, respectively, are:

$$
\begin{gathered}
\bar{R}=a \cos \hat{\phi} \\
\overline{\mathrm{I}}=a \sin \hat{\phi}
\end{gathered}
$$

Their variances are both equal to $\sigma_{0}^{2}$. The magnitude $\rho_{\mathrm{m}}$ of the voxel signal is found from:

$$
\begin{aligned}
\rho_{\mathrm{m}} & =\left(R^{2}+I^{2}\right)^{1 / 2} \\
& =\left(a^{2}+2 \eta_{1} a \cos \hat{\phi}+2 \eta_{2} a \sin \hat{\phi}+\eta_{1}^{2}+\eta_{2}^{2}\right)^{1 / 2} \\
& \simeq a\left(1+\frac{\eta_{1}}{a} \cos \hat{\phi}+\frac{\eta_{2}}{a} \sin \hat{\phi}\right)
\end{aligned}
$$

With the approximation made for $\rho_{\mathrm{m}}$ in Equation B6.1.36, the mean of the magnitude of the voxel signal is, as expected, given by:

$$
\overline{\rho_{\mathrm{m}}}=a
$$

To obtain the variance of the magnitude, the noise is taken to be uncorrelated between the real and imaginary channels. Then, from Equation B6.1.36:

$$
\begin{aligned}
\operatorname{var}\left(\rho_{m}\right) & \equiv \sigma_{\text {mag }}^{2} \\
& =\operatorname{var}\left(\eta_{2}\right) \cos ^{2} \hat{\phi}+\operatorname{var}\left(\eta_{1}\right) \sin ^{2} \hat{\phi} \\
& =\sigma_{0}^{2}
\end{aligned}
$$

i.e., if the SNR of the magnitude of the voxel signal is much larger than unity, the mean of the magnitude is the true magnitude value, and its variance is the same as the variance in either the real or imaginary part of the voxel signal.

\section{Measuring Noise in an Image}

Although it is tempting to simply measure the noise in the image itself, this can lead to overestimate of the noise. When there are structural changes in signal or when there is a nonuniform RF response, these variations in signal also lead to a variance in the measurement even when no noise is present. Only if the image is absolutely flat in the region-of-interest is it safe to do this. 
For this reason, the mean of the noise from the outside or background is used to estimate $\sigma_{0}$. It can be shown from the Raleigh distribution that this mean will be given by $\overline{\rho_{b}}=$ $1.253 \sigma_{0}$. Hence, by taking $0.8 \overline{\rho_{b}}$ from the background, we have an estimate for $\sigma_{0}$ as if we had taken it in the object itself. When you do this, make sure that you highlight a background region away from any ghosting artifact (usually place it in a region outside the object in the read direction as no ghosting is present there).

\section{Noise Variance in a Phase Image Inside the Object}

As before, the phase of the signal can be found from:

$$
\tan \hat{\phi}=I / R
$$

Using the approach of differentials on Equation B6.1.39 to determine the error gives:

$$
\delta \hat{\phi} \sec ^{2} \hat{\phi}=\frac{\delta I}{R}-\delta R \frac{I}{R^{2}}
$$

Hence, $\overline{\delta \hat{\phi}}=0$ and the variance of the phase is given by the mean squared error:

$$
\begin{aligned}
\overline{\delta \hat{\phi}^{2}} & =\left(\frac{I}{R}\right)^{2}\left(\frac{\overline{d I^{2}}}{I^{2}}+\frac{\overline{\delta R^{2}}}{R^{2}}\right) \cos ^{4} \hat{\phi} \\
& =\sigma_{0}^{2} \cos ^{4} \hat{\phi}\left(\frac{\rho_{\mathrm{m}}^{2}}{R^{4}}\right) \\
& =\frac{\sigma_{0}^{2}}{\rho_{\mathrm{m}}^{2}}
\end{aligned}
$$

and the standard deviation of the phase radians is:

$$
\sigma_{\text {phase }}=\left(\overline{\delta \phi^{2}}\right)^{1 / 2}=\frac{\sigma_{0}}{\rho_{\mathrm{m}}}
$$

or in degrees is:

$$
\sigma_{\text {phase }}=\frac{180^{\circ} \sigma_{0}}{\pi \rho_{\mathrm{m}}}
$$

This tells us that for voxels where the $\operatorname{SNR}\left(\rho_{\mathrm{m}} / \sigma_{0}\right)$ of the magnitude is very high, there is a relatively low error in phase measurements. On the other hand, the error is large if the voxel magnitude has low SNR.

\section{LITERATURE CITED}

Gudbjartson, H. and Patz, S. 1995. The Rician distribution of noisy MRI data. Magn. Reson. Med. 34:910-914.

Henkelman, R.M. 1985. Measurement of signal intensities in the presence of noise in MR images. Med. Phys. 12:232-233.

Kittel, C. 1969. Thermal Physics. John Wiley \& Sons, New York.

Papoulis. A. 1991. Probability, Random Variables, and Stochastic Processes. McGraw-Hill, New York.

Signal, Noise, and Contrast

B6.1.9 


\section{KEY REFERENCES}

Gudbjartson and Patz, 1995. See above.

This article provides a detailed discussion of noise in magnitude and phase images in MR.

Haacke, E.M., Brown, R.W., Thompson, M.R., and Venkatesan, R. 1999. Magnetic Resonance Imaging: Physical Principles and Sequence Design. John Wiley \& Sons, New York.

This text is an excellent reference on MR physics and covers most of the technical discussion presented in this unit.

Henkelman, 1985. See above.

Basic concepts of signal and noise in MR are well described in this paper.

Contributed by Azim Celik and Weili Lin

The University of North Carolina at Chapel Hill

Chapel Hill, North Carolina 